\title{
Optimization of Parameters of Wireless Sensor Network using Opportunistic Routing Algorithm
}

\author{
Ankita Bajpai ${ }^{1}$, Anamika Goswami ${ }^{2}$, Mahendra Kumar ${ }^{3}$ \\ M. Tech Scholar, Department of Electronics and Communication Engineering, B.I.E.T., Jhansi, U.P., India ${ }^{1,2}$ \\ Associate Professor, Department of Electronics and Communication Engineering, B.I.E.T., Jhansi, U.P., India ${ }^{3}$
}

\begin{abstract}
Savings optimization will become one of the principal worries in the wireless sensor community (WSN) routing protocol layout, because of the reality that maximum sensor nodes are geared up with the restrained nonrechargeable battery strength community lifetime for statistics relay. Following the principle of opportunistic routing idea, multi hop relay choice to optimize the network energy performance is made based totally at the differences amongst sensor nodes, in phrases of both their distance to sink and the residual strength of each different. In this paper, we propose a technique, which improves the Opportunistic routing algorithm on the basis of Energy, Network Lifetime, Delay and Delivery Ratio.
\end{abstract}

Keywords: ORARN, Delay, Energy, Routing, WSN.

\section{INTRODUCTION}

The WSN is usually a engineered of "nodes" - from a couple of number of nodes to many lots for may be thousands, where every node is connected to a minimum of one (or generally several) device. A device node may vary in size and energy from that of a box right down to the scale of a grain of salt, though functioning "speck" of real microscopic dimensions have however to be created. [1] The value of device nodes is equally variable, starting from a couple of to many green backs, betting on the quality of the individual device nodes. Size and price constraints on device nodes end in corresponding constraints on resources like energy, memory, procedure speed and communications information measure. [2][3]The topology of the WSNs will vary from a straightforward star network to a complicated multi-hop wireless mesh network. This paper is organized as follows: Section 1 gives brief introduction on WSN. Section 2 comprises of brief explanation of Opportunistic Routing algorithm for Relay Node Selection. The proposed technique and results are presented in Section 3. Section 4 presents conclusion of the paper.

\section{OPPORTUNISTIC ROUTING ALGORITHM}

Relay node choice in Opportunistic Routing concept is implemented for minimizing the energy intake and maximizing network life of a sensor community for static sensor nodes. this is completed to stability the network electricity performance through calculating the distance of the sensor nodes to the sink and the residual energy of every node. hence, an strength saving opportunistic routing principle is carried out to save the battery power and to enhance the network connectivity. Simulation consequences show that the proposed approach reveals accurate overall performance changes in electricity saving and network lifetime as compared with different present routing methodologies. [4]

\footnotetext{
Modules:

- Initialization

- Create forward set

- Relay node selection

- Communication
}

\section{Initialization:}

We remember a multi hop WSN; the relay node has lots of neighboring nodes. Nodes have some knowledge of the place records of their direct neighboring nodes and the position of the supply node and the sink node. each wireless sensor node has constant most transmission range $R$ and minimum transmission variety $d_{\min }$. Let $N(h)$ represents as the neighbor set of a node h, i.e., $n \in N(h)$. [1]

\section{Create forward set:}

The total energy dissipation in transmission and receiving required by node $\mathrm{h}$ to its neighboring node $\mathrm{n}$. energy consumption can be expressed as follows: [1] 


$$
\mathbf{E}_{\mathbf{T}}=\left(\mathbf{E}_{\text {elec }}+\boldsymbol{\varepsilon}_{\mathrm{amp}} \mathbf{d}^{\boldsymbol{\tau}}\right) \mathbf{B}
$$

On the other hand, the energy consumption of receiver ER can be calculated as follows

$$
\mathbf{E}_{\mathrm{R}}=\mathbf{E}_{\text {elec }} \mathbf{B}
$$

The total consumed energy $(\mathrm{Ch})$ of node $\mathrm{h}$ can be expressed as follows:

$$
\mathbf{C}_{\mathbf{h}}=\sum_{\mathrm{i}=\mathbf{0}}^{\mathbf{n}} \mathbf{E}_{\mathrm{T}}+\sum_{\mathrm{I}=\mathbf{0}}^{\mathbf{n}-\mathbf{1}} \mathbf{E}_{\mathbf{R}}
$$

Optimal transmission distance $d_{o p}$ for sensor node $h$ is

$$
d_{o p}=\frac{M-x_{h}}{n_{o p}}=\left\{\left(2 E_{\text {elec }}\right) /\left[(\tau-1) \varepsilon_{\text {amp }}\right]\right\}^{1 / \tau}
$$

This global minimum/maximum can be calculated as follows:

$$
\mathbf{n}_{\mathbf{o p}}=\frac{\left[(\tau-1) \varepsilon_{\text {amp }}\right]^{1 / \tau}\left(M-\mathbf{x}_{\mathbf{h}}\right)}{\left(2 \mathbf{E}_{\text {elec }}\right)^{1 / \tau}}
$$

Create the forward set for a node using the following lines.

For each node $\mathrm{i} \epsilon \mathrm{N}(\mathrm{h})$

$\operatorname{if}\left(\mathrm{d}\left(\mathrm{i}, \mathrm{d}_{\mathrm{op}}\right)<d\left(\mathrm{~h}, \mathrm{~d}_{\mathrm{op}}\right) \cup\left(\mathrm{E}_{\mathrm{i}}>\mathrm{c}\right)\right)$

Then add $i$ to $F(h)$.

\section{Relay node selection:}

We introduce ENS_OR algorithm for energy saving to select the next relay node which has the highest priority in forwarder set to forward the incoming ENS_OR algorithm.

For each node $i \in \mathrm{F}(\mathrm{h})$

$$
P(i)=\left(d_{i}-d_{h}\right)\left[\frac{1}{\left|d_{i}-d_{h}\right|}+\left(E_{i}-c\right)\right]
$$

\section{PROPOSED TECHNIQUE \& RESULTS}

We propose an energy-efficient routing algorithm for above 1-D queue network, particularly, energy saving via Opportunistic Routing (ENS_OR). ENS_OR choose the relay nodes based totally on opportunistic routing concept, to genuinely derive the most fulfilling transmission distance for electricity saving and maximizing the lifetime of complete community. Energy Saving through Opportunistic Routing (ENS_OR) best recall the power and priority parameter to select the relay node. We upload connectivity parameter with that parameter and advocate new power saving through Opportunistic Routing (ENS_OR) set of rules for higher transport ratio with lower postpone. Communication: the chosen source node ship data to their decided on relay node and the relay node forward their facts to their relay node until attain the vacation spot or base station.

\section{Simulation Results:}

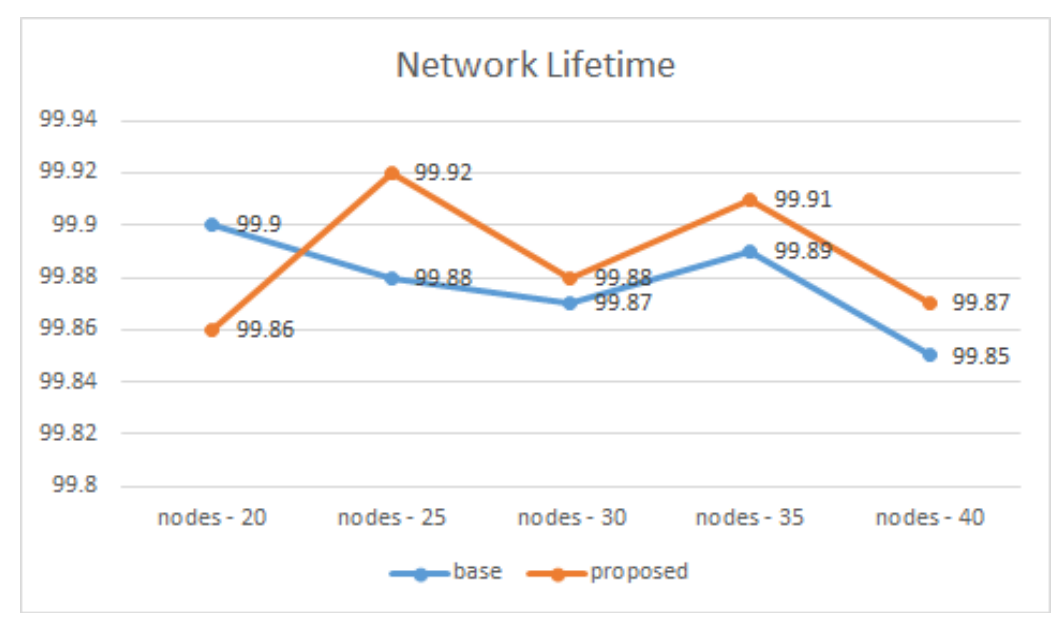

Figure 4.1: Comparison of Network Lifetime 
Figure 4.1 shows that Network Lifetime is increased in proposed technique when number of nodes is greater than 20 under simulation of such networks in NS2.

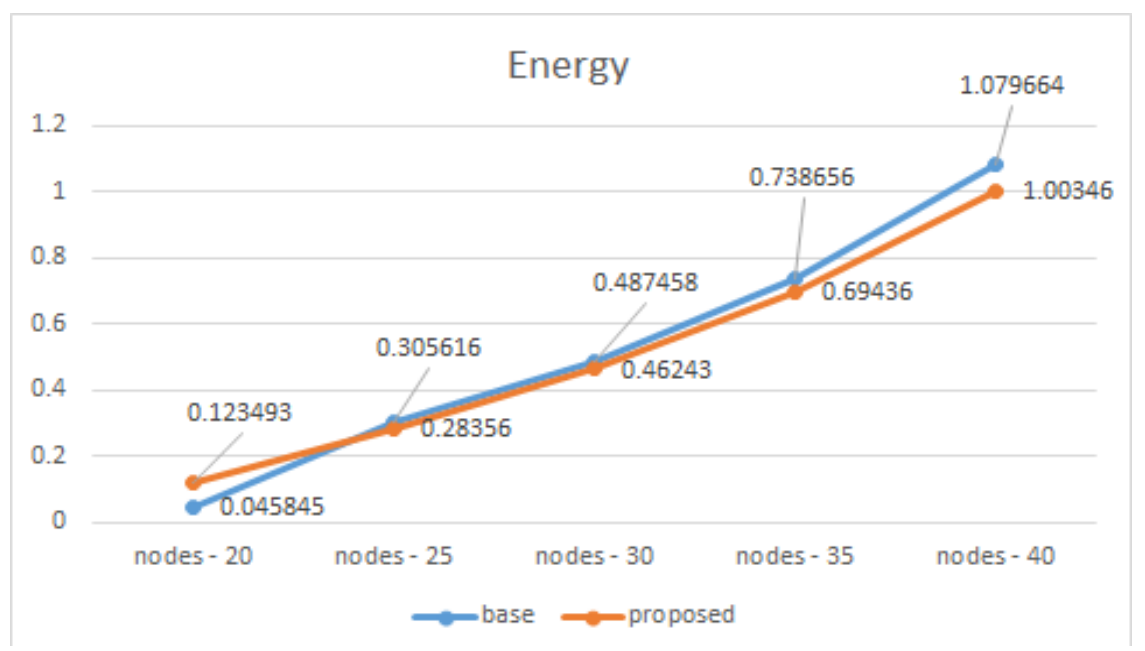

Figure 4.2: Comparison of Energy

Figure 4.2 shows that energy consumption is reduced in proposed technique.

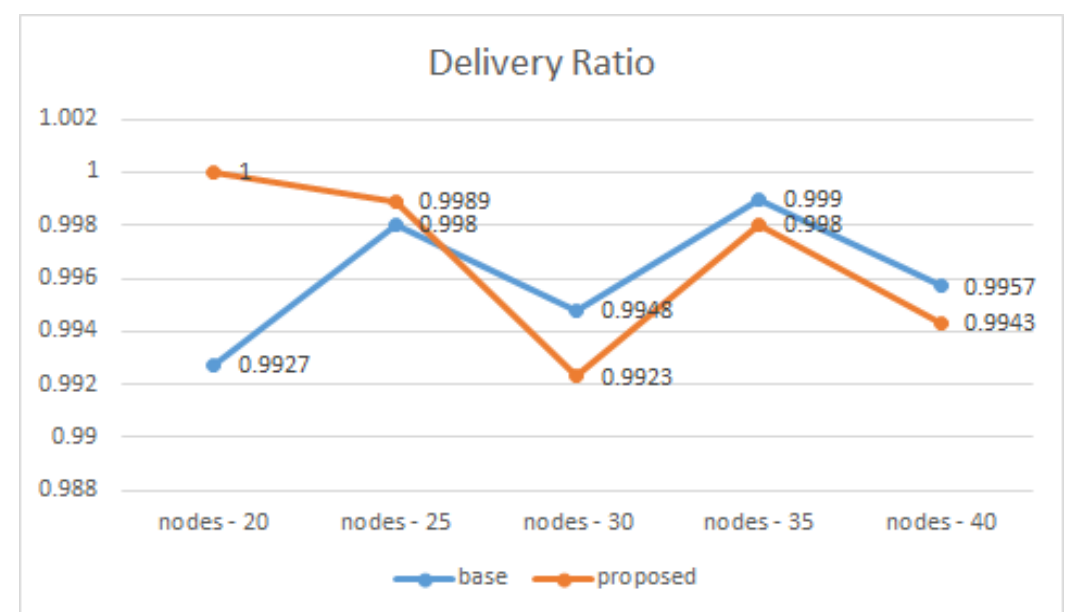

Figure 4.3: Comparison of Delivery Ratio

Figure 4.3 shows that delivery ratio is nearly the same in both the techniques, giving the ratio as 1 in 20 number of nodes in proposed technique.

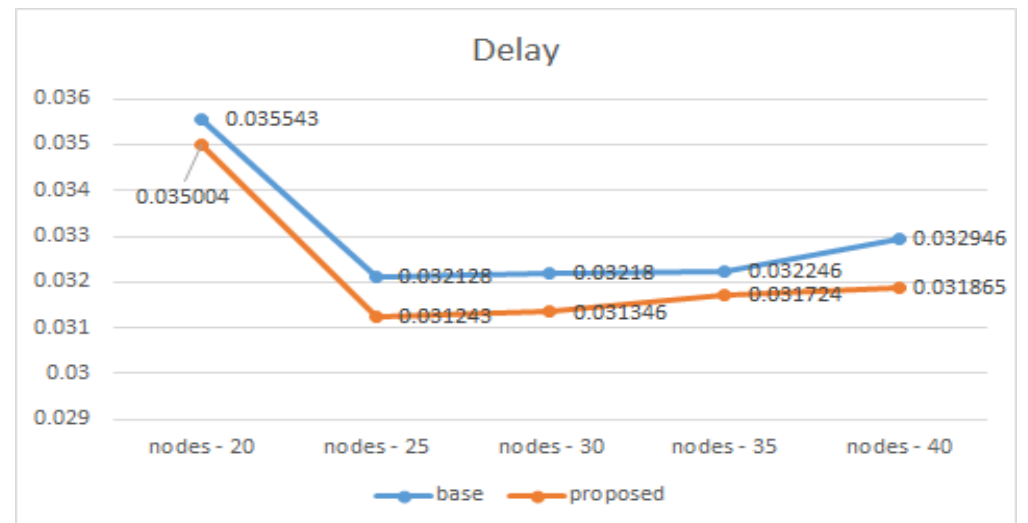

Figure 4.4: Comparison of Delay

Figure 4.4 clearly shows that in any number of nodes the delay is decreased in proposed technique. 


\section{CONCLUSION}

Opportunistic Routing in sensor networks is a brand new vicinity of studies, with a limited, however quick rising set of research consequences. on this paper, we presented a comprehensive survey of opportunistic routing strategies for WSNs. standard; the routing strategies are classified based at the community structure into 4 categories: information centric, hierarchical, place based totally and opportunistic routing protocols. We also speak numerous opportunistic routing protocols, and the design tradeoffs between strength and verbal exchange overhead financial savings in a number of the routing paradigm, with the advantages and drawbacks of each opportunistic routing technique. even though numerous of these opportunistic routing strategies appearance promising, there are nevertheless many demanding situations that want to be solved within the WSN. We highlighted those challenges and pinpointed destiny research directions on this regard.

\section{REFERENCES}

[1] Juan Luo, Member, IEEE, Jinyu Hu, Di Wu, Member, IEEE, and Renfa Li, Senior Member, IEEE, “Opportunistic Routing Algorithm for Relay Node Selection in Wireless Sensor Networks” IEEE TRANSACTIONS ON INDUSTRIAL INFORMATICS, VOL. 11, NO. 1, FEBRUARY 2015

[2] D. Bruckner, C. Picus, R. Velik,W. Herzner, and G. Zucker, "Hierarchical semantic processing architecture for smart sensors in surveillance networks,"IEEE Trans. Ind. Informat., vol. 8, no. 2, pp. 291-301, May 2012.

[3] L. LoBello and E. Toscano, "An adaptive approach to topology management in large and dense real-time wireless sensor networks," IEEE Trans. Ind. Informat., vol. 5, no. 3, pp. 314-324, Aug. 2009.

[4] D. Hoang, P. Yadav, R. Kumar, and S. Panda, "Real-time implementation of a harmony search algorithm-based clustering protocol for energy efficient wireless sensor networks,” IEEE Trans. Ind. Informat., vol. 10, no. 1, pp. 774-783, Feb. 2014

[5] F. Ren, J. Zhang, T. He, C. Lin, and S. K. Ren, "EBRP: Energy balanced routing protocol for data gathering in wireless sensor networks," IEEE Trans. Parallel Distrib. Syst., vol. 22, no. 12, pp. 2108-2125, Dec. 2011.

[6] A. Behnad and S. Nader-Esfahani, "On the statistics of MFR routing in one-dimensional ad hoc networks," IEEE Trans. Veh. Technol., vol. 60, no. 7, pp. 3276-3289, Sep. 2011.

[7] Shivleela. T, Dr. Anuradha M.S “Relay Node Selection Using Opportunistic Routing Algorithm in Wireless Sensor Network"International Journal of Electrical and Electronics Research ISSN 2348-6988 (online) Vol. 4, Issue 3, pp: (89-94), Month: July - September 2016

[8] J. Archana, Dr.V Bhanumathi," Relay Node Selection in Opportunistic Routing for Energy Saving in WSN" International Journal of Advanced Science and Engineering Research www.ijaser.in Volume: 1, Issue: 1, June 2016 